\title{
West Nile Virus Infection in the Mesopotamia Region, Syria Border of Turkey
}

\author{
Zehra Çağla Karakoç ${ }^{1}$, Bora Mete Tüzüner ${ }^{2}$, Onder Ergonul ${ }^{3}$, Anna Pierro ${ }^{4}$, Eugenio Di Fonzo ${ }^{4}$, \\ Ibrahim Koruk ${ }^{5}$, and Vittorio Sambri ${ }^{4}$
}

\begin{abstract}
We described the serological prevalence of West Nile Virus (WNV) antibodies among the human population in a historical and strategic region of Turkey. A serologic survey was conducted based on suspected cases in April, 2009, in the Mesopotamia region of Turkey, in the villages that were located alongside the Zergan River. All the sera were tested by enzyme-linked immunosorbent assay ELISA (Euroimmune ${ }^{\mathrm{TM}}$ ), and the positive samples were tested by immunofluorescent assay (IFA; Euroimmune ${ }^{\mathrm{TM}}$ ). As confirmation, neutralizing antibodies against WNV were tested by microneutralization assay (MNTA). In total, 307 individuals were included. The MNTA test was found to be positive among 52 individuals out of $307(17 \%)$. In multivariate analysis, age >50 [odds ratio $(\mathrm{OR})=5.2$, confidence interval $(\mathrm{CI}) 2.76-9.97, p<0.001)$ and being in an occupational risk group $(\mathrm{OR}=2.02$, CI $1.02-4.04, p=0.044)$ were found to be the risk factors for WNV seropositivity with the MNTA test. The physicians in the region should be aware of the risk of WNV infection and should be alerted to detect the clinical cases.
\end{abstract}

Key Words: West Nile virus—Turkey-Mesopotamia—Microneutralization assay.

\section{Introduction}

$\mathbf{W}$ EST Nile Virus (WNV) is a single-stranded RNA virus with a transmission cycle that involves primarily birds and mosquitoes. The main route of human infection is through the bite of an infected mosquito. The clinical symptoms among humans can vary from subclinical infection to an illness characterized by fever, malaise, anorexia, nausea, vomiting, diarrhea, headache, myalgia, and skin rash. Neurologic disease occurs in less than $1 \%$ of infected individuals (Vaughn et al. 2010).

In Turkey, the first serologic prevalence study of WNV infection among humans was performed in 1964 and serologic positivity was detected in different regions of the country (Heperkan and Ar1 1964). WNV infection in Turkey was reported in the 1970s based on the detection of hemagglutination-inhibiting antibodies (Meco 1977, Radda 1973). Acute WNV-infected cases were first detected in Aegean region of Turkey in 2010 (Kalaycioglu et al. 2012). The aim of the study was to assess the epidemiological status of the study area regarding WNV transmission in humans. The study area has global importance because of historical and strategic reasons.

\section{Materials and Method}

\section{Study population}

A serologic survey was conducted in April, 2009, following suspected cases with encephalitis that were detected about 6 months earlier in Kizıltepe region of Mardin province of Turkey. Kizıltepe is located at the southeastern part of Turkey, where it is called Mesopotamia (Fig. 1). This area is about $1403 \mathrm{~m}^{2}$, and the population is approximately 212,000 . The Zergan River, located in this region, is about $15 \mathrm{~km}$ long. The region has a semiarid climate with very hot and dry summers and cold, wet, and occasionally snowy winters. Temperatures in summer usually increase to $40^{\circ} \mathrm{C}-50^{\circ} \mathrm{C}$. Multiple reasons, such as socioeconomic factors, lack of hygiene and sanitation, and climatic features, provide a suitable environment for

\footnotetext{
${ }^{1}$ Florence Nightingale Hospital, Infectious Diseases and Clinical Microbiology, Şişli, Turkey.

${ }^{2}$ Istanbul Technical University, Department of Molecular Biology, Biotechnology and Genetics, Maslak, Turkey.

${ }^{3}$ Koç University, School of Medicine, Infectious Diseases and Clinical Microbiology Department, Istanbul, Turkey.

${ }^{4}$ Unit of Clinical Microbiology, Regional Reference Centre for Microbiological Emergencies (CRREM), St Orsola-Malpighi University Hospital, Italy.

${ }^{5}$ Harran University, Medical Faculty, Public Health Department, Şanlıurfa, Turkey.
} 


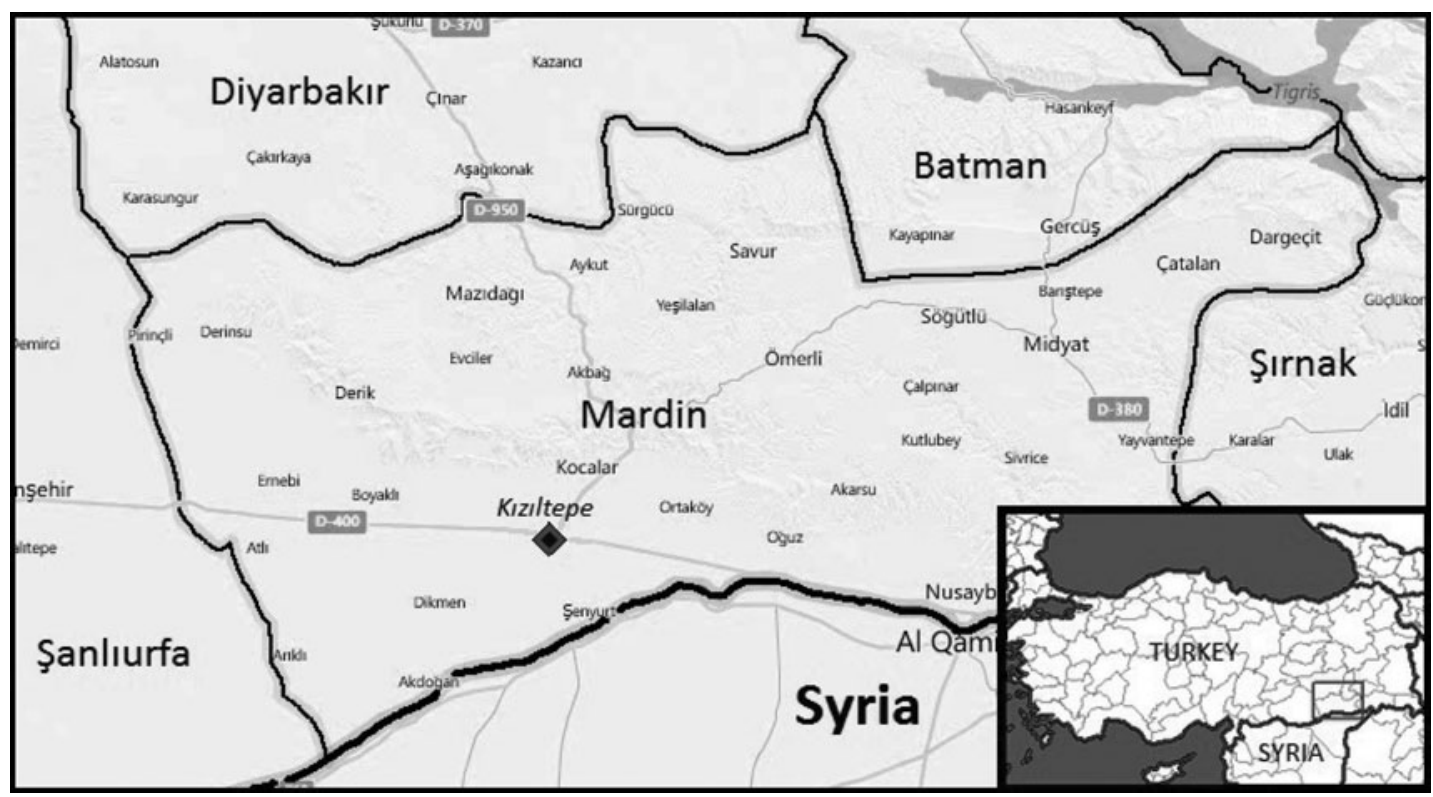

FIG. 1. The study area is located at the Syrian border.

epidemic infectious diseases in this region. In the region, 3009 people lived in nine villages with 365 houses and the villages neighboring Zergan River. The sample size calculation was performed based on the binomial probability of detecting at least one case. By estimating the minimum proportion of the serologic prevalence as $1 \%$ and the confidence intervals as $95 \%$, the sample size was calculated as 323 . The number of subjects $>16$ years of age to be included in the study were determined as proportional to the number of population of the villages. The houses were determined by randomization. One household member from each house was selected, and if there were more than one individual to be included from the house, one of the individuals was randomly selected. Among the selected 323 subjects, 307 were included in the study with a response rate of $95 \%$. The reasons not to be included in the study were not at home at the visit, migration for working, etc. Farmers, agricultural workers, unemployed people in the villages, and free traders, who had high potential to be exposed to the mosquitoes were categorized in high-risk occupation group, whereas housewives, teachers, students, and priests were grouped in low-risk occupation group. The survey form included the questions related to symptoms, such as fever, headache, flulike illness, rash, conjunctivitis, gastrointestinal complaints, and history of encephalitis. Besides the demographic information, potential risk factors such as occupation, socioeconomic conditions, living conditions, not using insect repellents, sleeping outside, and not using mosquito nets effectively were also questioned.

\section{Serologic testing}

The sera of the subjects were sent to Regional Reference Centre for Microbiological Emergencies, Clinical Microbiology Unit, St. Orsola-Malpighi University Hospital, Bologna, Italy. The steps for the process of virologic studies are depicted in Figure 2. All the sera were tested for WNV immunoglobulin $\mathrm{G}$ (IgG) by enzyme-linked immunosorbent assay (ELISA) (Euroimmun, Germany) and by an immuno- fluorescence assay (IFA) (Euroimmun, Germany). As confirmation, neutralizing antibodies against WNV were tested by a microneutralization assay (MNTA), as previously described (Capobianchi et al. 2010). An avidity test by IgG avidity assay (Euroimmun, Germany) (Fox et al. 2006) and titration of IgGseropositive samples by IFA were also performed. The samples with the presence of a titer >1:10 by MNTA were accepted as positive. If the MNTA titer against WNV was $\leq 1: 10$, sera were tested by MNTA against Usutu virus (USUV). If the MNTA titer against USUV was four-fold higher than MNTA titer against WNV, then samples were considered as USUV positive and WNV negative (Fig. 2). Samples were considered WNV positive if they tested positive on both ELISA and IFA, with positive confirmation by MNTA for WNV and not USUV; otherwise they were considered negative.

\section{Data analysis}

Data were analyzed using STATA Statistical Software, version 11.0 (College Station, TX). Mean comparisons for continuous variables were done using independent groups $t$ tests. Proportion comparisons for categorical variables were done by using chi-squared tests if data were sparse by the Fisher exact test. Multivariate analysis was performed for prediction of the risk of WNV infection. The outcome of the multivariate analysis was the WNV serologic positivity of the individuals. Statistically significant variables in univariate analysis, being in the occupational risk group and age $>50$ were included to the model as independent variables, and logistic regression was performed for prediction of WNV IgG positivity. The probability value for statistical significance was set as $<0.05$. The Mardin directorate of Health of Ministry of Health of Turkey approved the study.

\section{Results}

In total, 323 people from nine villages at the Syrian border of Turkey were targeted to be included to the study, and 307 


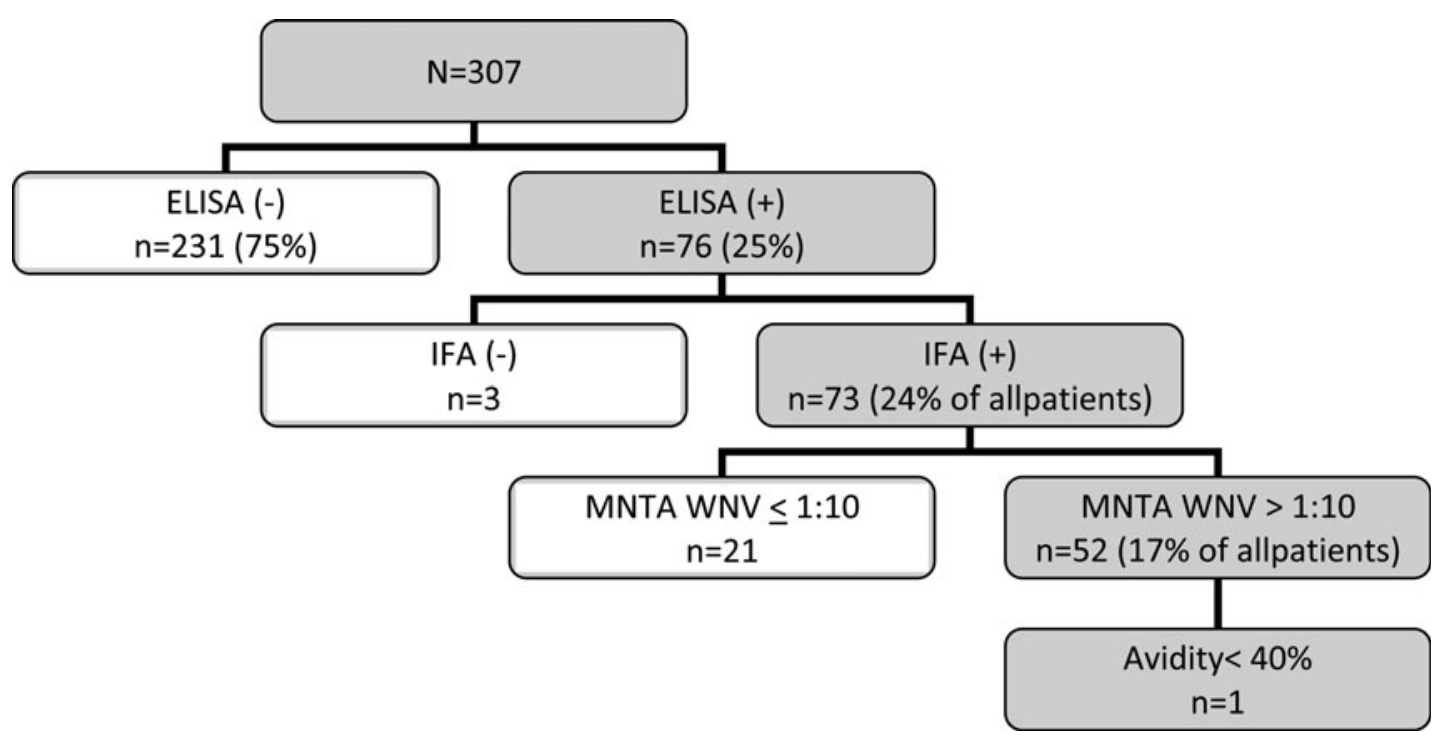

FIG. 2. The workflow of viral diagnosis of West Nile Virus infection.

individuals were successfully included. Sixteen people were not present at their addresses. WNV IgG positivity was detected in 76 individuals $(25 \%)$ by ELISA, and 73 out of 76 ELISA-positive samples were confirmed by IFA. Overall, WNV IgG positivity was $24 \%$ by IFA. In only one of the 76 seropositive individuals, the avidity test was $<40 \%$, indicating a recent infection. As confirmation, the MNTA test was found to be positive among 52 individuals out of 307 (17\%).

In univariate analysis, WNV serologic positivity by MNTA was found to be significantly associated with age $>50$ $(p<0.001)$. Among MNTA-positive individuals, the mean age was 52 [standard deviation (SD) 14], whereas among MNTAnegative individuals the mean age was 37 (SD 14). The serologic positivity among the people in the high-risk occupation group was found to be more statistically significant than the people in the low-risk occupation group (73\% versus $56 \%$, $p=0.026$ ) (Table 1 ). The parameters that could modify the exposure to WNV-infected mosquitoes, such as living conditions (air conditioning use, living in flats, and better protection, etc.), repellent use, sleeping outside, and using effective mosquito nets, were not statistically higher among the MNTA-positive group compared to the MNTA-negative group ( $p>0.05$; Table 1$)$. The serologic positivity in each village was $12.5 \%(n=40), 0(n=7), 0(n=6), 33 \%(n=6), 13 \%$ $(n=30), 25 \%(n=8), 0(n=6), 12 \%(n=17), 20 \%(n=187)$, and no significant difference was detected $(p=0.471)$.

There was no difference between MNTA-positive and MNTA-negative groups in terms of symptoms and signs within the last 6 months, such as fever, headache, flulike illness, and diarrhea (Table 1 ). In multivariate analysis, age $>50$ [odds ratio $(\mathrm{OR})=5.2$, confidence interval (CI) 2.76-9.97, $p<0.001]$ and being in an occupational risk group $(\mathrm{OR}=2.2$, CI 1.02-4.04, $p=0.044$ ) were found to be risk factors for WNV serologic positivity with MNTA test.

Table 1. Univariate Analyses of the Predictors for WNV Serologic Positivity

\begin{tabular}{|c|c|c|c|}
\hline & $\begin{array}{l}\operatorname{MNTA}(+) \\
\mathrm{n}=52(\%)\end{array}$ & $\begin{array}{l}\operatorname{MNTA}(-) \\
\mathrm{n}=255(\%)\end{array}$ & $\mathrm{p}$ \\
\hline Female gender & $27(52)$ & $151(59)$ & 0.332 \\
\hline Mean age (standard deviation) & $52(14)$ & $37(14)$ & $<0.001$ \\
\hline Age $>50$ & $27(52)$ & $43(17)$ & $<0.001$ \\
\hline Using repellents & $0(0)$ & $5(2)$ & 0.309 \\
\hline Sleeping outside & $52(100)$ & $251(98)$ & 0.363 \\
\hline Using effective mosquito nets & $1(2)$ & $10(4)$ & 0.480 \\
\hline \multicolumn{4}{|l|}{ Occupation } \\
\hline $\begin{array}{l}\text { Groups with higher risk to be exposed (includes farmers or agricultural } \\
\text { workers, unemployed, traders) }\end{array}$ & $38(73)$ & $144(56)$ & 0.026 \\
\hline $\begin{array}{l}\text { Groups with less risk to be exposed (includes housewives, teachers, } \\
\text { students, priests) }\end{array}$ & $14(27)$ & $111(44)$ & \\
\hline \multicolumn{4}{|l|}{ Symptoms and signs within the last year } \\
\hline Fever & $21(40)$ & $89(35)$ & 0.452 \\
\hline Headache & $14(27)$ & $69(27)$ & 0.984 \\
\hline Flu-like illness & $20(38)$ & $89(35)$ & 0.625 \\
\hline Gastrointestinal complaints including diarrhea & $4(8)$ & $20(8)$ & 0.971 \\
\hline
\end{tabular}

WNV, West Nile virus; MNTA, microneutralization assay. 


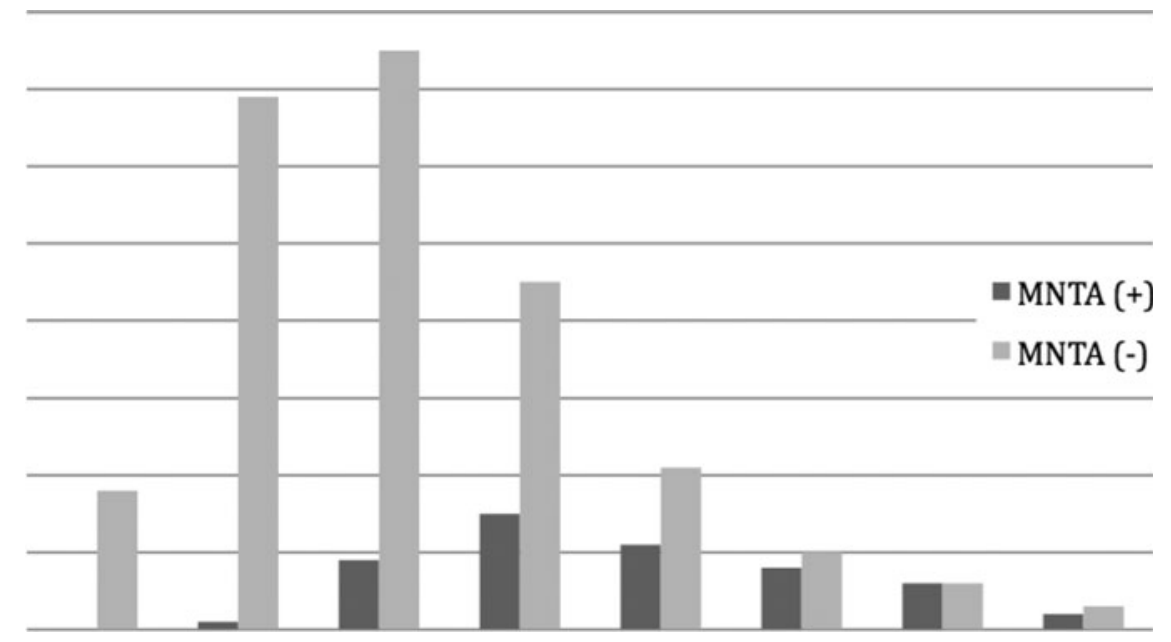

FIG. 3. Age distribution of microneutralization assay (MNTA) positive and negative subjects.

\section{Discussion}

This study was performed after detection of the suspected patients, who had signs of encephalitis. The diagnoses of these cases were not accurate because of the lack of appropriate conditions for viral studies to detect WNV in state hospitals. However, this study was performed to describe the extent of the infection in the region 6 months after detection of the suspected cases. We studied the Usutu virus of the Filaviviridae family to control the potential cross reaction. Using MNTA for confirmation increased the precision by decreasing the serologic positivity by ELISA and IFA tests.

The WNV positivity was found in $17 \%$ of the individuals, especially among higher age groups (Fig. 3). In multivariate analysis, the individuals with age $>50$ had five times more serologic positivity than the individuals with negative MNTA test $(\mathrm{OR}=5.2, \mathrm{CI} 2.76-9.97, p<0.001)$. The high level of serologic prevalence among the elderly in the region could be related to the past infections and outbreaks, although no case was reported. Meço performed a serologic prevalence study 35 years ago in the same region and the same season. He reported the serologic positivity for $\mathrm{WNV}$ as $48 \%$ (Meco 1977). However, he used a hemagglutination inhibition test as a method of detection. Ozer et al. performed a study among mosquitoes and the human population in the Şanlıurfa province, which neighbors the locality of our study. They reported $16 \%$ of WNV serologic positivity among 181 individuals who applied to the medical centers because of different reasons. They also studied the prevalence among 6547 mosquito specimens and did not detect WNV serologic positivity among mosquito specimens. They used the indirect immunofluorescence method and plaque reduction neutralization test for detection (Ozer et al. 2007).

The serologic positivity among the people in a high-risk occupation group was found to be higher than the people in the low-risk occupation group, which was statistically significant $(73 \%$ versus $27 \%, p=0.026$; Table 1$)$. In multivariate analysis, the individuals in the high-risk occupation group were found to have two times more MNTA positivity than the individuals in the lower-risk occupation group $(\mathrm{OR}=2.02, \mathrm{CI}$ $1.02-4.04, p=0.044)$. The parameters that could modify the exposure to the WNV-infected mosquitoes, such as repellent use, sleeping outside, and using effective mosquito nets, were not statistically different between MNTA-positive and MNTA-negative groups ( $p>0.05$; Table 1$)$. These risk factors were far from being detected because of the lack of sample size. However, being in a risk group because of occupation increased the likelihood of WNV serologic positivity.

There was no difference between MNTA-positive and MNTA-negative groups in terms of symptoms and signs within the last 6 months, such as fever, headache, flulike illness, and diarrhea (Table 1). After we performed this study, the first reported outbreak of WNV infection occurred in Turkey (Kalaycioglu et al. 2012). In August, 2010, 12 patients with the symptoms of fever, confusion, diarrhea, and rash were suspected of WNV infection. Four out of 12 patients were found to be positive for WNV with MNTA (Kalaycioglu et al. 2012). Although we did not detect acute cases in our study, the cases could be expected in the region. Our study in southeastern Anatolia and the outbreak in western Anatolia should alert all physicians to detect suspected cases of WNV infections.

\section{Conclusion}

High WNV serologic positivity (17\%) by MNTA was detected in the Mesopotamia region of Turkey, which is located at the Syria border. According to the MNTA test, all of the individuals except one had acquired the infection in the past. The individuals who have higher potential of exposure to the mosquitoes because of occupational risks were found to have higher serologic positivity for WNV infection $(\mathrm{OR}=2.05, \mathrm{CI} 1.02-4.13$, $p=0.043$ ). The physicians in the region should be aware of the risk of WNV infection and should be alerted to detect the clinical cases. An active health education program should be implemented to raise awareness about the disease in the region.

\section{Acknowledgments}

The authors thank the Mardin Directorate of Health, Hüseyin Nantu MD, Halil Kösesoy, Nermin Yalçın, Enise Irmak, Şahin Işınay, and Veysi Sertkaya for their help.

\section{Author Disclosure Statement}

No competing financial interests exist.

Author participation was as follows: Zehra Çağla Karakoç-field study, collection of data, official and ethics 
approval, manuscript preparation; Bora Tuzuner-laboratory work; Onder Ergonul-study design, data analysis, manuscript preparation; Anna Pierro-laboratory work; Eugenio Di Fonzo-laboratory work; Ibrahim Koruk-randomized selection of the subjects; Vittorio Sambri-laboratory work, manuscript preparation.

\section{References}

Capobianchi MR, Sambri V, Castilletti C, Pierro AM, et al. Retrospective screening of solid organ donors in Italy, 2009, reveals unpredicted circulation of West Nile virus. Euro Surveill 2010; 15.

Fox JL, Hazell SL, Tobler LH, Busch MP. Immunoglobulin G avidity in differentiation between early and late antibody responses to West Nile virus. Clin Vaccine Immunol 2006; 13: 33-36.

Heperkan Y, Arı A. Investigation of arboviruses in Turkey. Turk Hij Tecr Biyol Derg 1964; 24:113-118.

Kalaycioglu H, Korukluoglu G, Ozkul A, Oncul O, et al. Emergence of West Nile virus infections in humans in Turkey, 2010 to 2011. Euro Surveill 2012; 17.
Meco O. Investigation of West Nile virus specific haemagglutination inhibiting antibodies in southeastern Anatolian people. Mikrobiyol Bul 1977; 11:3-17.

Ozer N, Ergunay K, Simsek F, Kaynas S, et al. West Nile virus studies in the Sanliurfa Province of Turkey. J Vector Ecol 2007; 32:202-206.

Radda A. Studies on the activity and ecology of arboviruses in Turkey. Zentralbl Bakteriol. 1973; 225:19-26.

Vaughn DW, Barrett A, Solomon T. Flaviviruses. In: Mandell GL, Bennett JE, Dolin R, eds. Principles and Practice of Infectious Diseases. Philadelphia: Churchill Livingstone Elsevier; 2010: 2133-2156.

Address correspondence to: Onder Ergonul Department of Infectious Diseases Koç University Rumelifeneri Sariyer, Istanbul, 34266

Turkey

E-mail: oergonul@ku.edu.tr 